BMJ Open

Diabetes

Research

\& Care
To cite: Sørgjerd EP, Thorsby PM, Torjesen PA, et al. Presence of anti-GAD in a non-diabetic population of adults; time dynamics and clinical influence: results from the HUNT study. BMJ Open Diabetes Research and Care 2015;3:e000076. doi:10.1136/bmjdrc-2014000076

Received 18 December 2014 Revised 20 April 2015 Accepted 28 April 2015

This final article is available for use under the terms of the Creative Commons Attribution Non-Commercial 3.0 Licence; see http://drc.bmj.com

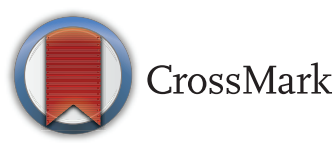

For numbered affiliations see end of article.

Correspondence to Dr Elin Pettersen Sørgjerd; elin.p.sorgjerd@ntnu.no

\title{
Presence of anti-GAD in a non-diabetic population of adults; time dynamics and clinical influence: results from the HUNT study
}

\author{
Elin Pettersen Sørgjerd, ${ }^{1}$ Per M Thorsby, ${ }^{2}$ Peter A Torjesen, ${ }^{2}$ Frank Skorpen, ${ }^{3}$ \\ Kirsti Kvaløy, ${ }^{1}$ Valdemar Grill ${ }^{4,5}$
}

\section{ABSTRACT}

Background: It is well known that anti-GAD (glutamic acid decarboxylase) serves as a marker for development of autoimmune diabetes in adults. On the other hand, the clinical implications of anti-GAD positivity in persistently non-diabetic (PND) adults are poorly elucidated. Our aim was to establish the frequency of anti-GAD in PNDs in an allpopulation-based cohort from the Nord-Trøndelag health study (HUNT) and to prospectively test for associations with glucose tolerance and thyroid autoimmunity.

Methods: We formed a primary study population (4496 individuals), selected randomly from the age group 20-90 years (50\% men/women), who were non-diabetic both at HUNT2 (1995-1997) and HUNT3 (2006-2008). Anti-GAD-positive individuals at HUNT2, together with anti-GAD-negative individuals aged 20-29 years, were retested for anti-GAD positivity at HUNT3. A secondary study population consisted of individuals with type 2 diabetes (T2D, n=349) at HUNT3 who developed diabetes between HUNT2 and HUNT3.

Results: The frequency of anti-GAD positivity in PND was $1.7 \%(n=76)$ at HUNT2. Positivity did not associate with gender, family history of diabetes, or glucose levels, but was associated with thyroidassociated autoimmunity (increased frequency of positivity for anti-TPO (thyroid peroxidase), $\mathrm{p}<0.002$ ). HLA-DQA1/DQB1, a risk haplotype for autoimmunity, was also associated with anti-GAD positivity in PND. The incidence of anti-GAD positivity was low $(0.4 \%)$ in the subsample of individuals who were anti-GAD negative in HUNT2. Anti-GAD positivity in PNDs was frequently evanescent, with $54 \%$ losing, usually lowgrade, positivity between HUNT2 and HUNT3.

An evanescent state of autoimmunity as assessed by anti-GAD positivity during "pre-diabetes" in individuals later diagnosed with T2D could, however, not be affirmed.

Conclusions: Anti-GAD positivity in PND is associated with HLA risk haplotypes and thyroid autoimmunity but not with clinical parameters of diabetes. Fleeting antiGAD positivity is common; however, results do not support the notion of a history of autoimmunity in T2D in the present cohort.

\section{INTRODUCTION}

Development of autoimmune diabetes is strongly associated with the presence of autoantibody markers and the most commonly used antibody for diagnostic purpose is glutamic acid decarboxylase (anti-GAD). This antibody is present in over $80 \%$ of patients with type 1 diabetes who develop disease during childhood or adolescence. ${ }^{1}$

Anti-GAD and other antibodies against pancreatic antigens can often be detected many years before the clinical onset of autoimmune-mediated diabetes, indicating a long "pre-diabetic" phase of autoimmune activity. $^{2}{ }^{3}$ Studies which identify individuals at risk of autoimmune diabetes by antibodies have mainly investigated relatives of patients with type 1 diabetes. ${ }^{4}{ }^{5}$ There are limited data available on antibody profiling in the general population. Prospective epidemiological data have shown that many individuals who develop autoimmune diabetes, in particular latent autoimmune diabetes in the adult (LADA), ${ }^{6}$ have antibodies before the onset of diabetes and then usually in conjunction with a family history of diabetes.

However, studies both in adults ${ }^{6-8}$ and in children $^{9}{ }^{10}$ have shown that antibodies, in particular anti-GAD, are also present in a proportion of non-diabetic individuals who do not develop diabetes over many years and who do not have close relatives with autoimmune diabetes. The frequency of anti-GAD positivity in such non-diabetic individuals varies between studies $(0.7-4.8 \%)$ and the clinical importance of positivity is debated. It has been argued that positivity under these conditions has no bearing on autoimmune diabetes, ${ }^{11} 12$ that it is unspecific (particularly if it is weak), or that it should be regarded as falsely positive. ${ }^{13}$ There is a scarcity of large and prospective epidemiological studies which are population-based and which 
specifically address the issue of persistently antibodypositive non-diabetic individuals.

The primary aim of the present study was to investigate the presence, persistence, and potential clinical implications of anti-GAD positivity in persistently nondiabetic adults. For this purpose, we prospectively analyzed relevant data in a large non-diabetic adult population-based cohort collected from the Norwegian Health Study in Nord-Trøndelag (HUNT). On finding positivity disappearing with time in many individuals, we also tested whether some antibody-negative individuals who were classified as having type 2 diabetes in the third HUNT survey could have displayed signs of autoimmunity (ie, anti-GAD positivity) prior to diabetes.

\section{MATERIAL AND METHODS \\ Study population}

The study is based on the second and third health surveys (HUNT2 and HUNT3) in Nord-Trøndelag county in Norway. HUNT2 was performed in 1995-1997 and HUNT3 in 2006-2008. Details about the HUNT study have been published previously. ${ }^{14}$ Briefly, the surveys represent $69 \%$ (HUNT2) and 54\% (HUNT3) of the entire adult population of Nord-Trøndelag county. For our analysis, we collected a random sample from 37059 adults $\geq 20$ years of age who took part in the HUNT2 and HUNT3 surveys, comprising about $40 \%$ of all adults in the county.

Equal numbers of men and women without diabetes, identified by self-reported answers of 'No' to the question "Do you have or have had diabetes?" at both surveys (in the following termed persistently non-diabetic individuals), were randomly selected from different age groups: 500 individuals aged 20-29, 500 aged 30-34, 500 aged 35-39, etc, up to the last age group with 500 individuals aged from 65 years and above. Altogether, we sampled a total of 4500 persistently non-diabetic individuals to represent the general adult population (figure 1). These individuals constituted our primary study population. Serum specimens from HUNT2 to be used for anti-GAD measurements were available for 4496 of the 4500 selected individuals. All individuals who were found to be anti-GAD positive in HUNT2 were analyzed for positivity in HUNT3. Additionally, we also tested for anti-GAD in HUNT3 in a subset of the anti-GAD-negative individuals. The subset comprised all randomly selected anti-GAD-negative individuals at HUNT2 who were 20-29 years of age at HUNT2. This age group was selected since age in HUNT3 corresponded with the 3039 year age group in HUNT2 which displayed the highest frequency of anti-GAD positivity.

We also included 53 adults with incident autoimmune diabetes from HUNT3 (reported not having diabetes in HUNT2 but reported having diabetes in HUNT3, ie individuals who developed diabetes between HUNT2 and HUNT3, figure 1) for comparison with our primary study population. Some results on these individuals have been published previously. ${ }^{2}$ Individuals were classified as having type 1 diabetes if they started insulin treatment within 12 months of diagnosis and were antibody positive or antibody negative, but in addition had fasting $\mathrm{C}$ peptide levels $<150 \mathrm{pmol} / \mathrm{L}$. Individuals were classified as having LADA if they were antibody positive at diagnosis and had not been treated with insulin within 12 months of diagnosis. No age limit was set for LADA. A subset of 32 of these 53 individuals was anti-GAD
Figure 1 Flow sheet over the primary study population as well as comparative individuals of "pre-diabetic" individuals followed from HUNT2 to HUNT3 (GAD, glutamic acid decarboxylase; neg, negative; pos, positive).

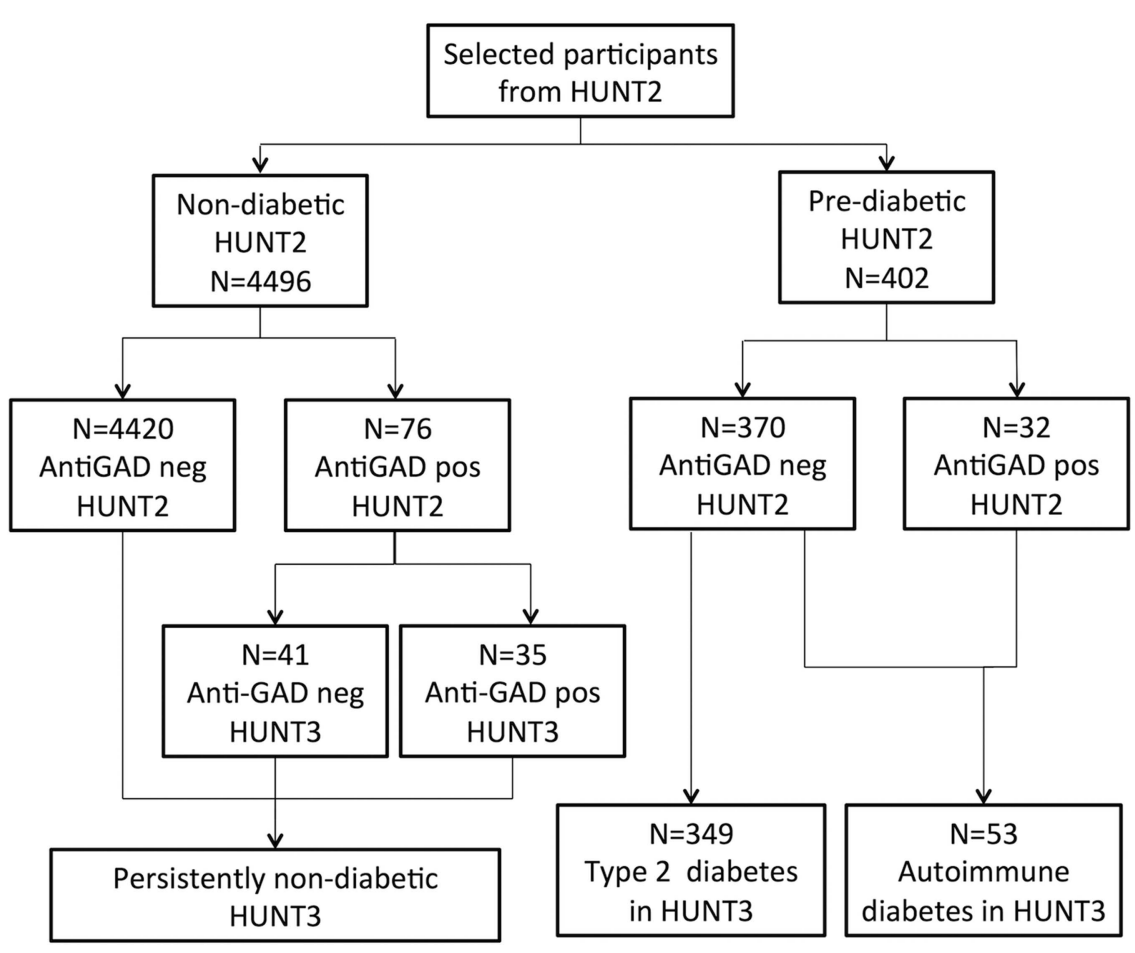


positive already at HUNT2 without a diagnosis of diabetes. Data from these 32 individuals who with time developed autoimmune diabetes and were anti-GAD positive prior to diabetes diagnosis, hereafter called autoimmune "pre-diabetic" individuals ("pre-diabetic" since we know that these individuals developed diabetes later), were compared with those from our primary study population who were anti-GAD positive and persistently non-diabetic.

To further study the time dynamics of anti-GAD in the HUNT cohort, we also investigated 349 individuals with incident type 2 diabetes in HUNT3, in whom anti-GAD could be analyzed in serum samples collected at HUNT2 (ie, years before diagnosis of diabetes). Classification of type 2 diabetes at HUNT3 was based on individuals being anti-GAD negative and not treated with insulin within 12 months of diagnosis.

\section{Antibody assays}

The serum samples used to analyze anti-GAD were stored at the HUNT Biobank, Levanger, Norway, at minus $80^{\circ} \mathrm{C}$ until analyzed. The samples were assayed for anti-GAD at the Hormone Laboratory, Department of Medical Biochemistry, Oslo University Hospital, Oslo, Norway. Anti-GAD was measured by immune precipitation using ${ }^{3} \mathrm{H}$ leucine translation-labeled GAD65 as the reagent (Novo Nordisk, Bagsværd, Denmark). Separation of bound anti-GAD and free labeled GAD65 was performed by protein $\mathrm{A}+\mathrm{C}$ coupled to Sepharose. Antibody levels were expressed as an antibody index (ai, method range $\geq 0.01 \mathrm{ai})$ relative to a standard serum given by the formula ((counts per minute (CPM) in the patient's sample-cpm from the negative reference sample)/(cpm of a positive reference sample-cpm from the negative reference sample)). We have used two different cut-offs for positivity, namely $\geq 0.06 \mathrm{ai}$ and $\geq 0.08$ ai. On the basis of the Hormone Laboratory's participation in the Diabetes Autoantibody Standardization Program (DASP), the cut-off of 0.06 ai gave a $82 \%$ DASP sensitivity and a 99\% DASP specificity (from the 2010 DASP), and the cut-off of 0.08 gave a $64 \%$ DASP sensitivity and a 100\% DASP specificity (from 2003 DASP). The intra-assay coefficient of variation (CV) was $14 \%$ in the lowest range (0.11ai), $8 \%$ in the middle range (0.22ai) and $17 \%$ in the highest range of measurements (2.0ai). The total CV was $19 \%$ in the lowest range (0.21ai) and $23 \%$ in the highest range of measurements (0.66ai).

Analysis of thyroid peroxidase antibody (anti-TPO) was implemented as a baseline measurement in HUNT2 and HUNT3. In HUNT2, anti-TPO was measured in all participants with measurements of thyroxin-stimulating hormone $(\mathrm{TSH})>4.0 \mathrm{mU} / \mathrm{L}$ and additionally in a $3 \%$ randomly selected group of participants of HUNT2. Measurements of anti-TPO were performed in nonfasting serum samples at the Hormone Laboratory, and were analyzed by luminous immunoassay technology. The instrument range was 201-9999 kIE/L and intra-assay CV was $11 \%$ in the low range $(214 \mathrm{kIE} / \mathrm{L})$,
$9 \%$ in the middle range $(412 \mathrm{kIE} / \mathrm{L})$, and $5 \%$ in the high range $(2300 \mathrm{kIE} / \mathrm{L})$. In HUNT3, anti-TPO was measured in all participants with measurements of $\mathrm{TSH}<0.10 \mathrm{mU} / \mathrm{L}$ or $\mathrm{TSH}>3.00 \mathrm{mU} / \mathrm{L}$. The measurements were performed in non-fasting serum at Levanger Hospital, Levanger, Norway, and were analyzed by chemiluminescent microparticle immunoassay technology. The instrument range was $0.01-1000 \mathrm{IU} / \mathrm{mL}$ and intra-assay $\mathrm{CV}$ was $18.5 \%$ in the low range $(0.7 \mathrm{IU} / \mathrm{mL})$ and $3.3 \%$ in the high range $(72.8 \mathrm{IU} / \mathrm{mL})$.

By combining results on anti-TPO from HUNT2 (positivity $\geq 200 \mathrm{kIE} / \mathrm{L}$ ) and HUNT3 (positivity $>5.1 \mathrm{IU} / \mathrm{mL}$ ), we obtained data on anti-TPO from 502 (mean age 60.6 \pm 13.2 and $42 \%$ men) of 4496 individuals.

\section{HLA typing}

Persistently non-diabetic individuals who were anti-GAD positive at HUNT2 as well as a control group of anti-GAD-negative non-diabetic individuals were typed for HLA-DQA1 and HLA-DQB1. The control group was age-matched and gender-matched to the anti-GADpositive, non-diabetic group. Two controls were selected per anti-GAD-positive individual.

HLA-DQA1 and HLA-DQB1 genotypes were analyzed at the Unit for Immunology, St. Olavs Hospital, Trondheim, Norway, by sequence-specific oligonucleotide probes using LABType ${ }^{\mathrm{R}}$ SSO DQA1/DQB1 Typing Tests (One Lambda Inc, California, USA), as described by the manufacturer. In brief, the target DNA was amplified by PCR using HLA-locus specific target primers. The amplified target DNA was denatured and rehybridized with complementary DNA probes bound to fluorescently coded microspheres, which allowed detection using R-Phycoerythrin-bound Streptavidin. The flow analyzer Luminex 100 (Luminex Corporation, Texas, USA) was used to measure the fluorescent signal of Phycoerythrin and the HLA fusion 2.0 (One Lambda Inc, California, USA) software was used to process the results.

The HLA DQA1-DQB1 haplotypes were divided into the following four groups based on known type 1 diabetes risk: (1) very high risk, having the genotype $D Q A 1 * 0301-D Q B 1 * 0302 / D Q A 1 * 0501-D Q B 1 * 0201$; high risk, having the genotypes $D Q A 1 * 0301-D Q B 1 * 0302 /$ $Z, \quad D Q A 1 * 0501-D Q B 1 * 0201 / Z$ or $D Q A 1 * X-D Q B 1 * 0302 /$ $D Q A 1 * X-D Q B 1 * X ;(3)$ neutral, having $D Q A 1 * X-D Q B 1 * X /$ $D Q A 1 * X-D Q B 1 * X ;(4)$ strongly protective, having either the genotype $D Q A 1 * X-D Q B 1 * 0602 / D Q A 1 * X-D Q B 1 * 0603$ or one of these haplotypes in combination with one of the haplotypes $D Q A 1 * X-D Q B 1{ }^{*} 0302$ or $D Q A 1{ }^{*} X-D Q B 1 * X$ (see table 3 for more details). For groups 2-4, $X$ indicates a non-defined allele and $\mathrm{Z}$ indicates any haplotype defined and non-defined except $D Q A 1 * 0301-D Q B 1 * 0302$ and $D Q A 1 * 0501-D Q B 1 * 0201$ (potential homozygosity was not excluded).

\section{Statistical analysis}

Data are given as numbers and percentage for categorical data and as median (minimum-maximum value) for 
continuous data. All statistical analyses were performed by the PASW Statistics (V.20, SPSS, Inc, Chicago, Illinois, USA). $\chi^{2}$ Test or Fisher's exact test (when appropriate) was used to compare differences in categorical data. The Mann-Whitney U test was used to test differences in continuous data between two groups. The Kruskal-Wallis test was used to test differences in continuous data between more than two groups. Logistic regression models were used to examine whether HLA haplotypes were associated with anti-GAD after adjusting for other confounding factors such as age, gender, body mass index (BMI), and first-degree family history of diabetes. A two-tailed $p$ value of 0.05 was considered to be significant.

\section{Ethics}

All participants gave their written consent. The study was approved by the Regional Committee for Ethics in Medical Research.

\section{RESULTS}

\section{Frequency of anti-GAD in persistently non-diabetic individuals}

Seventy-six of 4496 persistently non-diabetic individuals in HUNT3 (1.7\%) tested positive for anti-GAD in HUNT2 when using a cut-off of 0.06ai (median level 0.08 ai with minimum-maximum values of $0.06-3.58 \mathrm{ai}$ ). The age group with the highest frequency was 30-34 years (16 of $499,3.2 \%$ ). The age group with the lowest frequency was 45-49 years (2 of 499 individuals, $0.4 \%$, figure 2). There were no significant differences in frequency between sexes. When using 0.08ai as a cut-off for anti-GAD positivity, $41(0.9 \%)$ persistently non-diabetic individuals tested positive in HUNT2 (median level 0.23 ai with minimum-maximum values of $0.08-3.58 \mathrm{ai}$ ) In the following, the results are presented using the cut-off of 0.06 ai as anti-GAD positivity if not otherwise stated.

\section{Anti-GAD positivity in relation to clinical data}

Positivity for anti-GAD was not associated with smoking, non-fasting glucose, or BMI (table 1).

Nor was there an association with first-degree family history of diabetes (overall). When splitting first-degree family history of diabetes into parents, siblings, and children, there tended to be a higher risk of being anti-GAD positive in those who had siblings with diabetes (OR (95\% CI) 1.96 (0.83 to 4.51 ), $\mathrm{p}=0.14,8.2 \%$ (6 of 73 individuals) vs $4.4 \%$ (190 of 4292 individuals)).

\section{Anti-GAD disappearance with time}

Of the 76 initially anti-GAD-positive adults in HUNT2, a majority, that is, $41(53.9 \%)$, converted to negative in the follow-up sample in HUNT3. There were no phenotypic differences between persistently anti-GAD-positive individuals compared with transiently anti-GAD-positive individuals (table 2). However, the initial anti-GAD levels in HUNT2 was higher in persisters compared with converters, with a median level (minimum-maximum value) of 0.27 ai $(0.06-3.58 \mathrm{ai})$ versus $0.06 \mathrm{ai}(0.06-1.34 \mathrm{ai}) \quad(\mathrm{p}<0.01$ for difference).

\section{Cumulative incidence of anti-GAD in an initially anti-GAD-negative population subset}

Of the initially 495 anti-GAD-negative adults in the age group 20-29 years at HUNT2, we could sample 485

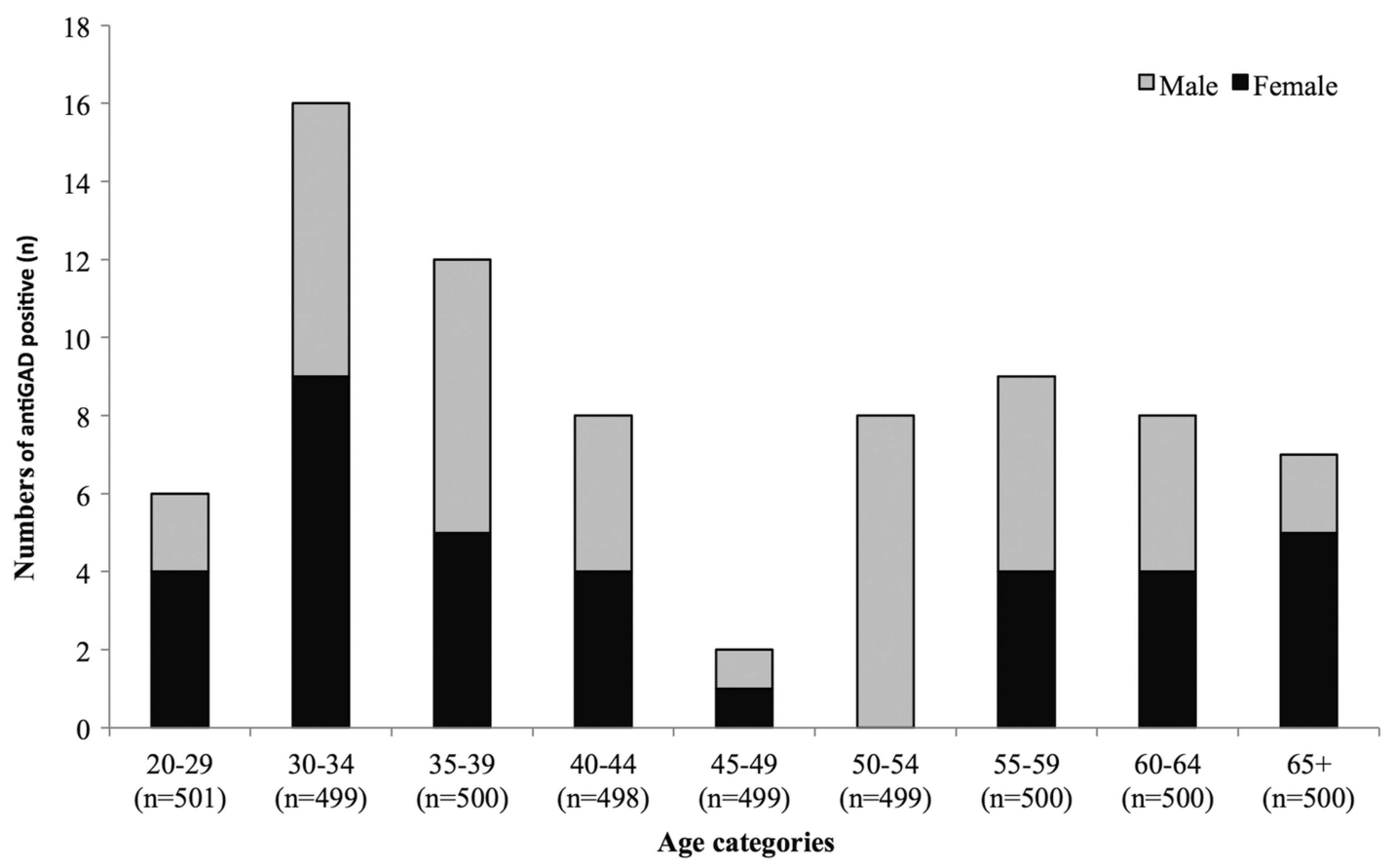

Figure 2 Frequency of anti-GAD (glutamic acid decarboxylase) positivity in the adult population of the HUNT2 survey distributed across different age categories. 
Table 1 Clinical characteristics of persistently non-diabetic individuals stratified by being anti-GAD negative and anti-GAD positive in HUNT2

\begin{tabular}{|c|c|c|c|}
\hline & Anti-GAD negative & Anti-GAD positive & p Value \\
\hline $\mathrm{N}$ & 4420 & $76(1.7 \%)$ & \\
\hline Sex (male) & $2208(50.0 \%)$ & $40(52.6 \%)$ & 0.64 \\
\hline Age at attendance & $48(19-85)$ & $42(21-77)$ & 0.22 \\
\hline Waist circumference (cm) & $86(55-131)$ & $84(64-109)$ & 0.69 \\
\hline BMI $\left(\mathrm{kg} / \mathrm{m}^{2}\right)$ & $25.6(16.2-52.8)$ & $26.0(19.4-34.8)$ & 0.70 \\
\hline Systolic blood pressure (mm Hg) & $133(82-225)$ & 132 (102-189) & 0.82 \\
\hline Diastolic blood pressure $(\mathrm{mm} \mathrm{Hg})$ & 79 (44-136) & $78(52-120)$ & 0.85 \\
\hline Glucose, non-fasting ( $\mathrm{mmol} / \mathrm{L})$ & $5.1(2.6-14.4)$ & $5.1(3.5-7.5)$ & 0.89 \\
\hline Cholesterol (mmol/L) & $5.8(1.9-13.2)$ & $5.7(3.4-8.2)$ & 0.35 \\
\hline HDL cholesterol (mmol/L) & $1.4(0.5-3.8)$ & $1.3(0.6-2.4)$ & 0.62 \\
\hline Triacylglycerol (mmol/L) & $1.4(0.3-18.6)$ & $1.3(0.2-4.7)$ & 0.30 \\
\hline \multicolumn{4}{|l|}{ Family history of diabetes } \\
\hline No & 3229 & 55 & \\
\hline Yes & $1181(26.8 \%)$ & $21(27.6 \%)$ & 0.86 \\
\hline \multicolumn{4}{|l|}{ Smoking } \\
\hline No & 3224 & 59 & \\
\hline Yes & $1052(24.9 \%)$ & 17 (22.4\%) & 0.65 \\
\hline
\end{tabular}

Data are given as numbers (\%) and median (minimum-maximum value).

Unadjusted $p$ value calculated by the Mann-Whitney $U$ test for continuous data and by the $\chi^{2}$ test for categorical data.

BMI, body mass index; GAD, glutamic acid decarboxylase; HDL, high-density lipoprotein.

serum samples from HUNT3. These samples were then analyzed for anti-GAD positivity. Only two individuals were found to be positive (0.06ai and 0.42ai), giving an 11-year cumulative incidence of $0.4 \%$.

\section{Anti-GAD-positive non-diabetic persisters display less diabetogenic traits than anti-GAD-positive autoimmune "pre-diabetic" individuals}

We compared (table 2) persistently non-diabetic individuals who were anti-GAD positive at HUNT2 $(n=76)$ with autoimmune "pre-diabetic" individuals who were anti-GAD positive and non-diabetic at HUNT2 but had developed diabetes prior to or at HUNT3 $(n=32)$. Actual values of anti-GAD in HUNT2 and HUNT3 of persistent non-diabetic (divided into persistently and transiently anti-GAD positive) versus autoimmune 'pre-diabetic' individuals are shown in online supplementary figure S1.

Autoimmune "pre-diabetic" individuals displayed a higher frequency of first-degree family history of diabetes compared with those who remained non-diabetic $(\mathrm{p}<0.01)$. This difference was also significant after adjusting (by logistic regression) for HLA genotypes, sex, age, and BMI (OR 3.24 (1.22 to 8.62), $\mathrm{p}=0.02$ ). Autoimmune "pre-diabetic" individuals at HUNT2 also displayed significantly higher anti-GAD levels $(\mathrm{p}<0.01)$ and non-fasting glucose levels $(p<0.01)$. In addition, they were more obese (higher waist circumference and BMI, $p=0.01$ and $p=0.02$, respectively) compared with the persistently non-diabetic individuals. Removing those of the latter who became anti-GAD negative in HUNT3 $(n=41)$ from the analysis did not alter the differences between the persistently non-diabetic and autoimmune "pre-diabetic" individuals (data not shown).

\section{No evidence for fleeting autoimmunity during}

\section{"pre-diabetes" in type 2 diabetic individuals}

On finding a major and unexpected rate of disappearance of anti-GAD positivity from HUNT2 to HUNT3, we reasoned that a similar process could possibly include a "pre-diabetic" period of fleeting autoimmune activity in individuals who are later diagnosed with type 2 diabetes on the basis of negative testing for autoimmune antibodies. To exclude such a clinically important possibility, we sampled 349 serum samples from HUNT2 adults who reported not having diabetes in HUNT2 but who were classified as having type 2 diabetes in HUNT3. None of these individuals were found to be anti-GAD positive in the "pre-diabetic" state (HUNT2).

\section{Anti-GAD positivity in persistently non-diabetic individuals associates with HLA risk haplotypes}

Seventy-five anti-GAD-positive and 151 anti-GAD-negative persistently non-diabetic individuals were successfully genotyped for HLA DQA1-DQB1. We found no differences regarding the clinical characteristics when comparing the anti-GAD-positive and anti-GAD-negative groups (data not shown). However, having very high-risk or high-risk HLA genotypes was associated with being anti-GAD positive (table 3) after adjusting for age, sex, BMI, and first-degree family history of diabetes (OR (95\% CI) 9.57 (2.16 to 42.16$), \mathrm{p}<0.01$ and OR 2.21 (1.02 to 4.77 ), $\mathrm{p}=0.04$, respectively).

Removing the converters (ie, those who became anti-GAD negative in HUNT3, $\mathrm{n}=41$ ) from the analysis did not materially affect the results (data not shown). Comparing only the converters with the anti-GADnegative ones showed a trend for associations between 
Table 2 Clinical characteristics from HUNT2 of (1) anti-GAD-positive and initially non-diabetic individuals at HUNT2 (stated to be not having diabetes), divided into

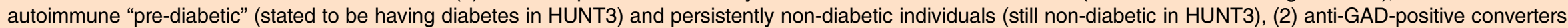
and persisters measured in HUNT3 in persistently non-diabetic participants

\begin{tabular}{|c|c|c|c|}
\hline $\begin{array}{l}\text { (1) Autoimmune } \\
\text { "pre-diabetic"* } \\
\text { Anti-GAD positive } \\
\text { HUNT2 }\end{array}$ & $\begin{array}{l}\text { (1) Persistently } \\
\text { non-diabetic } \\
\text { Anti-GAD positive } \\
\text { HUNT2 }\end{array}$ & $\begin{array}{l}\mathbf{p} \\
\text { Value }\end{array}$ & $\begin{array}{l}\text { (2) Persistently non-diabetic } \\
\text { converters§ } \\
\text { Anti-GAD negative HUNT3 }\end{array}$ \\
\hline
\end{tabular}

(2) Persistently non-diabetic

32

$\mathrm{N}$

32

Sex (male)

Age at attendance $\quad 50(26-76)$

Waist circumference $(\mathrm{cm})$

$40(52.6 \%)$

$42(21-77)$

$84(64-109)$

$92(65-109)$

BMl $\left(\mathrm{kg} / \mathrm{m}^{2}\right)$

$27.8(20.9-39.6)$

Systolic blood pressure $(\mathrm{mm} \mathrm{Hg}) \quad 137(107-190)$

$26.0(19.4-34.8)$

Diastolic blood pressure $(\mathrm{mm} \mathrm{Hg}) \quad 85(58-122)$

$131(102-189)$

$78(53-120)$

$5.1(3.5-7.1)$

$5.7(3.4-8.2)$

Cholesterol $(\mathrm{mmol} / \mathrm{L})$

$5.5(4.1-10.0)$

$6.0(3.4-7.6)$

$1.3(0.6-2.4)$

HDL cholesterol $(\mathrm{mmol} / \mathrm{L})$

$1.3(0.7-2.4)$

$1.6(0.6-7.8)$

$1.44(0.07-1.98)$

$1.3(0.2-4.7)$

Anti-GAD level (ai)

Family history of diabetes

$18(56.3 \%)$

$0.08(0.06-3.58)$

41

persisters |

Smoking (currently)

$12(37.5 \%)$

$21(27.6 \%)$

$17(22.4 \%)$

$\begin{array}{rc}1.00 & 19(46.3 \%) \\ 0.19 & 44(22-77) \\ 0.01 & 82(64-120) \\ 0.02 & 25.6(19.4-34.2) \\ 0.05 & 131(102-188) \\ 0.05 & 78(60-120) \\ <0.01 & 5.2(3.5-7.5) \\ 0.22 & 5.5(3.4-8.2) \\ 0.24 & 1.3(0.7-2.4) \\ 0.13 & 1.3(0.2-4.0) \\ <0.01 & 0.06(0.06-1.34) \\ <0.01 & 12(29.3 \%) \\ 0.15 & 11(26.8 \%)\end{array}$

HUNT3

p

35

Data are presented as $n(\%)$ or median (minimum-maximum values).

*Autoimmune "pre-diabetic"; individuals having autoimmune diabetes in HUNT3 (type 1 diabetes $(n=13)$ and LADA ( $n=21)$ ), but reported not having diabetes and did not have non-fasting glucose $<11 \mathrm{mmol} / \mathrm{l}$ in HUNT2.

†Persistently non-diabetic; individuals who were non-diabetic both at HUNT2 and HUNT3.

fUnadjusted $p$ value calculated by the Mann-Whitney $U$ test for continuous data and by Fisher's exact test for categorical data. Comparison between anti-GAD-positive autoimmune "pre-diabetic" and persistently non-diabetic individuals.

§Persistently non-diabetic converters; anti-GAD positive in HUNT2 and converted to being negative in HUNT3.

IPersistently non-diabetic persisters; anti-GAD positive in HUNT2 and HUNT3.

**Unadjusted $\mathrm{p}$ value calculated by the Mann-Whitney $U$ test for continuous data and by Fisher's exact test for categorical data. Comparison between anti-GAD-positive converters and persisters among the persistently non-diabetic individuals.

ai, Antibody index; BMI, body mass index; GAD, glutamic acid decarboxylase; HDL, high-density lipoprotein; LADA, latent autoimmune diabetes in adult. 
Table 3 Association of the HLA DQA1-DQB1 haplotypes compared between anti-GAD-negative and anti-GAD-positive persistently non-diabetic individuals in HUNT2

\begin{tabular}{|c|c|c|c|c|c|c|}
\hline & \multirow{2}{*}{$\begin{array}{l}\text { Haplotype } 1 \\
\text { HLA } \\
\text { DQA1-DQB1 }\end{array}$} & \multirow{2}{*}{$\begin{array}{l}\text { Haplotype } 2 \\
\text { HLA } \\
\text { DQA1-DQB1 }\end{array}$} & \multicolumn{2}{|l|}{ Anti-GAD } & \multirow[b]{2}{*}{ OR (95\% Cl) } & \multirow[b]{2}{*}{ p Value } \\
\hline & & & Negative $\mathrm{N}=151$ & Positive $\mathbf{N}=75$ & & \\
\hline Very high risk & 0301-0302 & 0501-0201 & $3(2.0)$ & $8(10.7)$ & 9.57 (2.16 to 42.16$)$ & $<0.01$ \\
\hline High risk & $\begin{array}{l}0301-0302 \\
0501-0201 \\
X^{*}-0302\end{array}$ & $\begin{array}{l}\mathrm{Z} \dagger \\
\mathrm{Z} \\
\mathrm{X}-\mathrm{X}\end{array}$ & $61(40.4)$ & 37 (49.3) & 2.21 (1.02 to 4.77$)$ & 0.04 \\
\hline Neutral & $X-X$ & $X-X$ & $45(29.8)$ & $18(24.0)$ & $1.46(0.62$ to 3.40$)$ & 0.39 \\
\hline Strong protective & $\begin{array}{l}X-0602 \\
X-0602 \\
X-0602 \\
X-0603 \\
X-0603 \\
X-0603\end{array}$ & $\begin{array}{l}X-0603 \\
X-0302 \\
X-X \\
X-0602 \\
X-0302 \\
X-X\end{array}$ & $42(27.8)$ & $12(16.0)$ & 1.00 & \\
\hline
\end{tabular}

anti-GAD positivity and HLA risk haplotypes (very highrisk OR 5.51 ( 0.72 to 42.26$)$, $\mathrm{p}=0.10$, high-risk OR 2.14 (0.77 to 5.93), $\mathrm{p}=0.14$ and neutral OR 2.27 (0.79 to $6.54), \mathrm{p}=0.13$ ) after adjusting for age, sex, BMI, and firstdegree family history of diabetes.

\section{Anti-GAD positivity in persistently non-diabetic individuals associates with thyroid autoimmunity}

We tested if anti-GAD positivity was associated with antiTPO positivity using data from 502 individuals with measures on both. Within the anti-GAD positive individuals $(n=14), 78.6 \%$ were also anti-TPO positive $(n=11)$. In contrast, of the anti-GAD negative individuals $(n=488)$, only $36.7 \%$ were anti-TPO positive $(n=179) \quad(p<0.01$ for difference). There were no other clinical differences (as listed in table 1) between the anti-GAD positive and anti-GAD negative individuals within this subgroup (data for subgroup not shown).

\section{Results analyzed by a higher cut-off (0.08ai)}

Anti-GAD positivity was still not associated with clinical parameters of diabetes when using 0.08ai as a cut-off. Seroconversion from HUNT2 to HUNT3 decreased to about one-third (29.3\%). There were still no phenotypic differences between persisters compared with converters, and converters had lower anti-GAD levels. Significant differences between persistently non-diabetic individuals compared with autoimmune "pre-diabetic" individuals regarding parameters such as first-degree family history, anti-GAD, and non-fasting glucose levels were upheld (data not shown). Association between anti-GAD positivity and HLA risk haplotypes tended to be stronger when using 0.08ai as a cut-off for positivity (data not shown). Five of $7(71.4 \%)$ anti-GAD-positive individuals were also anti-TPO positive compared with 181 of $495(36.6 \%)$ anti-GAD-negative ones $(p=0.11)$.

\section{DISCUSSION}

In persistently non-diabetic individuals from a population-based cohort from Norway with 11 years of follow-up, we found a frequency of anti-GAD positivity of $1.7 \%$. We found no signs of reduced glucose tolerance in these anti-GAD-positive individuals, but there were still associations with high-risk type 1 diabetes HLA genotypes as well as with thyroid autoimmunity. Another observation was the appreciable loss of positivity with time. This finding opened the possibility of fleeting positivity during the "pre-diabetes" state in patients classified as type 2 diabetes. This possibility, based on measurements of anti-GAD in such patients, could, however, not be affirmed.

Including autoimmune "pre-diabetic" individuals together with the persistently non-diabetic individuals the frequency of anti-GAD positivity increases from $1.7 \%$ to $2.0 \%$. This frequency is in fair agreement with most previous reports in which frequencies range from $1 \%$ to $4 \%$ in children and adults from the general population. ${ }^{5} 6815$ In previous reports, a distinction was usually not made as to whether diabetes developed over time or not, nor to phenotypic characteristics and age as described here. Here, the individuals studied were sampled in a randomized and representative fashion to encompass different ages and both sexes. From these data, it seems clear that prevalent positivity was not overrepresented to a major extent by one sex, nor completely confined to certain ages.

The incidence of anti-GAD in our population was tested in a subset of our population, that is, those being 
20-29 years old and anti-GAD negative in HUNT2. Only two of these individuals developed positivity when retested at HUNT3, that is, 11 years later, whereas there was a much higher (2-3\%) positivity among HUNT2 prevalent individuals in the corresponding age groups. Perhaps then, the frequency data reflect to a large extent positivity that developed before the age of 20 years and then persisted. Such a notion must, however, be confirmed by other studies.

In the persistently non-diabetic individuals, we could not detect any clinical parameters conferring risk of diabetes (listed in table 1) that were associated with anti-GAD positivity. Neither did the presence or absence of stability of anti-GAD positivity (positivity or not both in HUNT2 and HUNT3) affect the clinical parameters. However, having high-risk HLA DQA1-DQB1 haplotypes were associated with the presence of anti-GAD. This is in line with a recent study mainly based on adult relatives of individuals with type 2 diabetes reporting that anti-GAD was also associated with HLA high-risk haplotypes in the absence of a family history of type 1 diabetes and evolution of diabetes. ${ }^{16}$

The notion that even a minor and fleeting anti-GAD positivity is coupled to autoimmunity is reinforced by finding associations between anti-GAD positivity and thyroid-associated autoimmunity. Hence, we found a clear association of the presence of antibodies with TPO. Whereas an association between type 1 diabetes as well as LADA and thyroid autoimmune disease is well documented, ${ }^{17-19}$ an association with the presence of anti-GAD in persistently non-diabetic individuals has, to the best of our knowledge, not been previously reported.

Fleeting anti-GAD positivity raises the possibility that individuals who had developed diabetes between HUNT2 and HUNT3 and were classified as type 2 could have been anti-GAD positive before diabetes diagnosis. If so, they would belong to the category of LADA rather than type 2 diabetes. This clinically important possibility was contradicted by the lack of anti-GAD positivity in this group. However, we cannot exclude the possibility of fleeting positivity for other antibodies in a subset of patients classified as type 2 diabetes.

We compared two different cut-offs for anti-GAD positivity and found that they gave essentially the same results. This finding is a further indication that the positivity for anti-GAD that we find in persistently nondiabetic individuals is not a statistical artifact.

A comparison of anti-GAD-positive persistently nondiabetic individuals with autoimmune "pre-diabetic" individuals (ie, anti-GAD positive, but non-diabetes at HUNT2, diabetes diagnosis at HUNT3) revealed higher titers of anti-GAD in HUNT2 in the latter. This could signify higher autoimmune activity, which in turn could precipitate overt diabetes in these individuals. However, we note that risk factors for type 2 diabetes such as BMI and age were also higher in the autoimmune "prediabetic" individuals and could participate in the development of diabetes.
In contrast to the Botnia study, ${ }^{16}$ we did not find an association between anti-GAD positivity and first-degree family history of diabetes in the non-diabetic individuals. This difference between studies may be explained by our questionnaire data which did not distinguish between type 1 and type 2 family histories of diabetes. The risk of developing type 1 diabetes and LADA is reportedly highest in siblings of patients with diabetes. $^{20}{ }^{21}$ We did see a tendency for higher risk of being anti-GAD positive in those individuals who had siblings with diabetes.

There are limitations to be considered when interpreting the results of this study. The number of anti-GAD-positive individuals that we detected and analyzed was moderate (although large compared with previous studies), something which precluded a detailed analysis of the influence of age and sex. Comparing anti-GAD positivity with $\mathrm{C}$ peptide levels would have added valuable data; however, the lack of fasting blood samples made such comparisons impossible. That data on thyroid autoimmunity are based on a subset of our primary population is another limitation. Confirming results from other populations would be desirable. A limitation by number also pertains to our incidence data (only subset analysis was possible). As to methodology, one may argue that anti-GAD positivity should be confirmed by competition assays. However, the latter type of analysis in a previous study did not detect any unspecific influence between three different anti-GAD assays. ${ }^{22}$ Stability of the anti-GAD assay is supported by validation against DASP. However, ideally, the intraindividual variation of levels of anti-GAD should have been ascertained by repeat measurements in a time perspective of weeks or months. Such repeated measurements would have added confirmation of the correctness of the classification into positivity or non-positivity for anti-GAD.

We conclude that anti-GAD positivity in adult nondiabetic individuals from the general population is not restricted in terms of age and sex, is only partly

\section{Key messages}

Anti-GAD can be detected many years before the clinical onset of autoimmune diabetes, which indicate a long pre-diabetic phase of autoimmune activity. The presence and clinical implications of anti-GAD positivity in persistently non-diabetic adults are, however, poorly elucidated.

- Using the population from the second and third NordTrøndelag Health Study, Norway, we found a frequency of anti-GAD positivity of $1.7 \%$ in a sub-group of persistently non-diabetic individuals.

- Our results show that there are no associations between antiGAD positivity and clinical parameters related to diabetes in non-diabetic adults. However, anti-GAD positivity is associated with high-risk HLA haplotypes linked to type 1 diabetes and also thyroid autoimmunity. 
persistent over a long time period, and is not associated with clinical parameters related to diabetes. However, such positivity is associated with increased frequency of high-risk HLA haplotypes linked to type 1 diabetes and also with thyroid autoimmunity. Further, fleeting anti-GAD positivity during "pre-diabetes" does not seem to be a factor behind the later development of diabetes which is diagnosed as type 2 diabetes.

\section{Author affiliations}

${ }^{1}$ Department of Public Health and General Practice, Faculty of Medicine, HUNT Research Centre, The Norwegian University of Science and Technology, Levanger, Norway

${ }^{2}$ Hormone Laboratory, Department of Medical Biochemistry, Oslo University Hospital, Oslo, Norway

${ }^{3}$ Department of Laboratory Medicine, Children's and Women's Health, Faculty of Medicine, The Norwegian University of Science and Technology,

Trondheim, Norway

${ }^{4}$ Department of Cancer Research and Molecular Medicine, Faculty of Medicine, The Norwegian University of Science and Technology, Trondheim, Norway

${ }^{5}$ Department of Endocrinology, St. Olav University Hospital, Trondheim, Norway

Acknowledgements The HUNT study was performed as a collaboration between the HUNT Research Centre (Faculty of Medicine, Norwegian University of Science and Technology (NTNU)), Nord-Trøndelag County Council, Central Norway Health Authority, and the Norwegian Institute of Public Health. The authors would like to thank Ms Oddrun Storrø, Ms Marit Aarhaug, and Ms Anne Kristin Lysakerrud at the Unit for Immunology at St. Olavs Hospital, Trondheim, Norway; and Ms Lisbeth B Kraft at Hormone Laboratory, Oslo University Hospital, Oslo, Norway, for their appreciated technical assistance.

Contributors EPS carried out the primary analysis and interpretation of the data and wrote the manuscript. PMT and PAT were involved in the anti-GAD analysis and reviewed/edited the manuscript. FS and KK were involved in the interpretation of data and reviewed/edited the manuscript. VG designed the study and was involved in the interpretation of data, writing and reviewing the manuscript. EPS, FS, KK and VG were involved in the acquisition of funding. All the authors gave their final approval of the article to be published.

Funding Our study was funded by the liaison committee of the Central Norway Regional Health Authority and the NTNU, the liaison committee of St. Olav's Hospital Trust and the Faculty of Medicine, NTNU, and from the Research Funds of St. Olav Hospital, Trondheim, Norway.

Competing interests None declared.

Patient consent Obtained.

Ethics approval The Regional Committee for Ethics in Medical Research.

Provenance and peer review Not commissioned; externally peer reviewed.

Data sharing statement We used data from the HUNT study. These data are available to anyone who applies to HUNT to get an authorization to use it.

Open Access This is an Open Access article distributed in accordance with the Creative Commons Attribution Non Commercial (CC BY-NC 4.0) license, which permits others to distribute, remix, adapt, build upon this work noncommercially, and license their derivative works on different terms, provided the original work is properly cited and the use is non-commercial. See: http:// creativecommons.org/licenses/by-nc/4.0/

\section{REFERENCES}

1. Baekkeskov S, Aanstoot HJ, Christgau S, et al. Identification of the $64 \mathrm{~K}$ autoantigen in insulin-dependent diabetes as the GABA-synthesizing enzyme glutamic acid decarboxylase. Nature 1990;347:151-6.

2. Sorgjerd EP, Skorpen F, Kvaloy K, et al. Time dynamics of autoantibodies are coupled to phenotypes and add to the heterogeneity of autoimmune diabetes in adults: the HUNT study, Norway. Diabetologia 2012;55:1310-18.

3. Tuomilehto J, Zimmet P, Mackay IR, et al. Antibodies to glutamic acid decarboxylase as predictors of insulin-dependent diabetes mellitus before clinical onset of disease. Lancet 1994;343:1383-5.

4. Vermeulen I, Weets I, Costa O, et al. An important minority of prediabetic first-degree relatives of type 1 diabetic patients derives from seroconversion to persistent autoantibody positivity after 10 years of age. Diabetologia 2012;55:413-20.

5. Siljander HT, Veijola R, Reunanen A, et al. Prediction of type diabetes among siblings of affected children and in the general population. Diabetologia 2007;50:2272-5.

6. Lundgren VM, Isomaa B, Lyssenko V, et al. GAD antibody positivity predicts type 2 diabetes in an adult population. Diabetes 2010;59:416-22.

7. Rolandsson O, Hagg E, Janer M, et al. High GAD65 autoantibody levels in nondiabetic adults are associated with HLA but not with CTLA-4 or INS VNTR. J Intern Med 2003;253:447-53.

8. Ruige JB, Batstra MR, Aanstoot $\mathrm{HJ}$, et al. Low prevalence of antibodies to GAD65 in a 50- to 74-year-old general Dutch population. The Hoorn Study. Diabetes Care 1997;20:1108-10.

9. LaGasse JM, Brantley MS, Leech NJ, et al. Successful prospective prediction of type 1 diabetes in schoolchildren through multiple defined autoantibodies: an 8-year follow-up of the Washington State Diabetes Prediction Study. Diabetes Care 2002;25:505-11.

10. Schlosser M, Strebelow M, Rjasanowski I, et al. Prevalence of diabetes-associated autoantibodies in schoolchildren: the Karlsburg Type 1 Diabetes Risk Study. Ann N Y Acad Sci 2004;1037:114-17.

11. Rolandsson $\mathrm{O}$, Hagg $\mathrm{E}$, Nilsson $\mathrm{M}$, et al. Prediction of diabetes with body mass index, oral glucose tolerance test and islet cell autoantibodies in a regional population. J Intern Med 2001;249:279-88.

12. Bosi EP, Garancini MP, Poggiali F, et al. Low prevalence of islet autoimmunity in adult diabetes and low predictive value of islet autoantibodies in the general adult population of northern Italy. Diabetologia 1999;42:840-4.

13. Gale EA. Latent autoimmune diabetes in adults: a guide for the perplexed. Diabetologia 2005;48:2195-9.

14. Holmen J, Midthjell K, Krüger $\varnothing$, et al. The Nord-Trøndelag Health Study 1995-97 (HUNT2). Objectives, contents, methods and participation. Nor J Epidemiol 2003;13:19-32.

15. Knip M, Korhonen S, Kulmala P, et al. Prediction of type 1 diabetes in the general population. Diabetes Care 2010;33:1206-12.

16. Andersen MK, Lundgren $\mathrm{V}$, Isomaa $\mathrm{B}$, et al. Association of variants in HLA-DQA1-DQB1, PTPN22, INS, and CTLA4 with GAD autoantibodies and insulin secretion in nondiabetic adults of the Botnia Prospective Study. Eur J Endocrinol 2012;167:27-33.

17. Hansen D, Bennedbaek FN, Hansen LK, et al. Thyroid function, morphology and autoimmunity in young patients with insulin-dependent diabetes mellitus. Eur J Endocrinol 1999;140:512-18.

18. Barova H, Perusicova J, Hill M, et al. Anti-GAD-positive patients with type 1 diabetes mellitus have higher prevalence of autoimmune thyroiditis than anti-GAD-negative patients with type 1 and type 2 diabetes mellitus. Physiol Res 2004;53:279-86.

19. Kordonouri O, Charpentier N, Hartmann R. GADA positivity at onset of type 1 diabetes is a risk factor for the development of autoimmune thyroiditis. Pediatr Diabetes 2011;12:31-3.

20. Carlsson S, Midthjell K, Grill V. Influence of family history of diabetes on incidence and prevalence of latent autoimmune diabetes of the adult: results from the Nord-Trøndelag Health Study. Diabetes Care 2007;30:3040-5.

21. Steck AK, Rewers MJ. Genetics of type 1 diabetes. Clin Chem 2011;57:176-85.

22. Daka B, Svensson MK, Lernmark K, et al. Low agreement between radio binding assays in analyzing glutamic acid decarboxylase (GAD65Ab) autoantibodies in patients classified with type 2 diabetes. Autoimmunity 2009;42:507-14 